\title{
GRID MODIFICATION \\ FOR SECOND-ORDER HYPERBOLIC PROBLEMS
}

\author{
DAOQI YANG
}

\begin{abstract}
A family of Galerkin finite element methods is presented to accurately and efficiently solve the wave equation that includes sharp propagating wave fronts. The new methodology involves different finite element discretizations at different time levels; thus, at any time level, relatively coarse grids can be applied in regions where the solution changes smoothly while finer grids can be employed near wave fronts. The change of grid from time step to time step need not be continuous, and the number of grid points at different time levels can be arbitrarily different. The formulation is applicable to general second-order hyperbolic equations. Stability results are proved and a priori error estimates are established for several boundary conditions. Our error estimates consist of three parts: the time finite difference discretization error, the spatial finite element discretization error, and the error due to the projections of the approximated solution from old grids onto new grids.
\end{abstract}

\section{INTRODUCTION}

In dealing with steep wave fronts using finite element methods $[3,8,10,14$, 16 ] one needs to apply very fine grids in order to reduce numerical oscillation, since error components that are nonoscillatory with respect to a fine grid are usually oscillatory with respect to a coarser grid. However, wave fronts often represent a small fraction of the physical domain and a uniformly refined grid over the whole domain results in large-size linear systems of algebraic equations. This may be computationally expensive, or even impossible to solve, owing to the limited capacity of computer memory. Thus it is desirable to apply relatively coarse grids in regions where the solution changes slowly and finer grids in regions where wave fronts occur.

When wave fronts propagate, one needs to be assured that the refined grid areas vary with the wave fronts. This requires the finite element method having the capability of discretizing the domain differently at different times. This should differ from the moving finite element method $[2,4,11,17,18]$ since the latter requires (basically) a fixed number of grid points at all time levels and a continuous change in grid. In [24], the author proposed a dynamic finite element method for the wave equation which can apply arbitrary grids at

Received by the editor June 4, 1993 and, in revised form, October 10, 1994.

1991 Mathematics Subject Classification. Primary 65M60, 65M50, 65M12.

Key words and phrases. Dynamic finite element methods, Galerkin methods, grid modification, second-order hyperbolic problems.

This work was supported in part by the Purdue Research Foundation. 
any time level (as long as the minimum-angle property of the triangulation is satisfied). There, an equivalent first-order system, both in space and in time, replaced the second-order wave equation, and was approximated by two mixed finite element schemes. Unconditional stability was proved and optimal error estimates were obtained. However, the method in [24] does not seem to apply to more general second-order hyperbolic equations.

In this paper we present a family of Galerkin finite element schemes which are applicable to nonlinear second-order hyperbolic equations. These schemes have the desired property that they allow one to employ dynamic finite elements in order to efficiently and accurately resolve sharp moving wave fronts; the grid at a time level can be arbitrarily different from that at the previous time level and need not change continuously in any fashion, as opposed to the moving finite element method. This property is especially desirable for problems in which an initial wave pulse splits into left- and right-going waves at later instances of time, since a fixed number of finite elements at all time levels cannot work well in this case. Also, these schemes have optimal rates of convergence, and many of them are unconditionally stable.

For brevity and clarity, only linear problems will be considered in this paper. Nonlinear problems can be treated analogously by the argument presented herein plus the techniques found in the papers by Wheeler [22] and the author [23]. Also, our previous paper [24] will be cited in order to shorten the argument.

In $\S 2$ we shall pose the differential problem and summarize some known results. Then, in $\S 3$, we shall define our numerical schemes of grid modification and give stability results. In $\S 4$, we prove the error estimates. Finally, in $\S 5$, we make some remarks.

\section{NOTATION AND PRELIMINARIES}

Suppose that $\Omega$ is a smooth bounded domain or a convex polygon in $\mathbb{R}^{d}$ with boundary $\Gamma$, and that $\rho(x), a(x) \in L^{\infty}(\Omega)$ are bounded above and below by positive constants on $\bar{\Omega}=\Omega \cup \Gamma$. Also suppose that $u(x, t)$ satisfies

$$
\begin{gathered}
\rho(x) \frac{\partial^{2} u}{\partial t^{2}}-\nabla \cdot(a(x) \nabla u)=f(x, t), \quad(x, t) \in \Omega \times J, \\
u(x, 0)=u_{0}(x), \quad \frac{\partial u}{\partial t}(x, 0)=v_{0}(x), \quad x \in \Omega,
\end{gathered}
$$

where $J=(0, T]$ with $T$ being a positive constant. The boundary condition satisfied by $u$ is taken to be

$$
u(x, t)=0, \quad(x, t) \in \Gamma \times J,
$$

or

$$
-a(x) \frac{\partial u}{\partial \nu}=g(x, t)+a_{1}(x) u+a_{2}(x) \frac{\partial u}{\partial t}, \quad(x, t) \in \Gamma \times J,
$$

where $a_{1}, a_{2}$ are nonnegative functions and $\frac{\partial}{\partial \nu}$ is outward normal differentiation.

We shall let $H^{p}(\Omega)$ and $H^{p}(\Gamma)$ denote the usual Sobolev spaces on $\Omega$ and $\Gamma$ for any real number $p$. The norm on $H^{p}(\Omega)$ will be denoted by $\|\cdot\|_{p}$ and 
that on $H^{p}(\Gamma)$ by $|\cdot|_{p}$; the subscript will be omitted when $p=0$. However, the weighted $L^{2}$ norm in $L^{2}(\Omega)$ with weight function, e.g., $\rho$, will be denoted by $\|\cdot\|_{\rho}$, and that in $L^{2}(\Gamma)$ by $|\cdot|_{\rho}$.

We assume that $a_{1}$ and $a_{2}$ are sufficiently regular that multiplication by $a_{1}$ or $a_{2}$ is a continuous operation on $H^{\frac{1}{2}}(\Gamma)$; a sufficient condition is that $a_{1}$ and $a_{2}$ are in $H^{1+\epsilon}(\Gamma)$ for $d=1,2,3$ and any $\epsilon>0$ [9]. Note that $(2.2 \mathrm{~b})$ is a general boundary condition of which first-order absorbing boundary conditions $[7,12,21]$ are special cases.

The standard way to solve the problem (2.1) and (2.2a) is as follows. First define a variational formulation: find $u(t) \in H_{0}^{1}(\Omega)$ with $u_{t t} \in L^{2}(\Omega)$ such that for $t \in J$

$$
\begin{gathered}
\left(\rho \frac{\partial^{2} u}{\partial t^{2}}, w\right)+(a \nabla u, \nabla w)=(f, w), \quad \forall w \in H_{0}^{1}(\Omega), \\
u(0)=u_{0}, \quad \frac{\partial u}{\partial t}(0)=v_{0},
\end{gathered}
$$

where

$$
(f, g)=\int_{\Omega} f \cdot g d x .
$$

Let $S_{h} \subset H_{0}^{1}(\Omega)$ be a finite element space. Then the well-known semidiscrete scheme is defined as follows: find $U(t) \in S_{h}$ such that for $t \in J$

$$
\begin{gathered}
\left(\rho \frac{\partial^{2} U}{\partial t^{2}}, w\right)+(a \nabla U, \nabla w)=(f, w), \quad \forall w \in S_{h}, \\
U(0)=U_{0}, \quad \frac{\partial U}{\partial t}(0)=V_{0},
\end{gathered}
$$

where $U_{0}, V_{0} \in S_{h}$ are some approximations of the initial data $u_{0}, v_{0}$, respectively.

The problem (2.4) is equivalent to the following system of ordinary differential equations: find $\xi(t) \in \mathbb{R}^{m}$ satisfying

$$
\begin{gathered}
B \frac{d^{2} \xi}{d t^{2}}+A \xi=F, \\
\xi(0)=\theta_{0}, \quad \frac{d \xi}{d t}(0)=\theta_{1},
\end{gathered}
$$

where $\xi, \theta_{0}$, and $\theta_{1}$ are respectively the coordinates of $U, U_{0}, V_{0}$ with respect to the basis functions $\left\{\phi_{1}, \phi_{2}, \ldots, \phi_{m}\right\}$ of $S_{h}, A=\left(\left(a \nabla \phi_{i}, \nabla \phi_{j}\right)\right)$ and $B=\left(\left(\rho \phi_{i}, \phi_{j}\right)\right)$ are $m \times m$ matrices, and $F=\left[\left(f, \phi_{1}\right), \ldots,\left(f, \phi_{m}\right)\right]^{T}$ is the force vector.

To discretize the problem (2.5) in time, we partition $J$ as $0=t_{0}<t_{1}<$ $\cdots<T_{N}=T$, and let $\eta=\frac{d \xi}{d t}$. Denote the approximations of $\xi\left(t_{n}\right)$ and $\eta\left(t_{n}\right)$ by $\xi_{n}$ and $\eta_{n}$, respectively. Then the fully discrete scheme reads: find $\xi_{n+1}, \eta_{n+1} \in \mathbb{R}^{m}$ such that

$$
B \frac{\eta_{n+1}-\eta_{n}}{\Delta t_{n}}+\alpha A \xi_{n+1}+(1-\alpha) A \xi_{n}=\alpha F_{n+1}+(1-\alpha) F_{n},
$$




$$
\begin{gathered}
\frac{\xi_{n+1}-\xi_{n}}{\Delta t_{n}}-\left(\beta \eta_{n+1}+(1-\beta) \eta_{n}\right)=0, \quad n=0,1, \ldots, N-1, \\
\xi_{0}=\theta_{0}, \quad \eta_{0}=\theta_{1},
\end{gathered}
$$

where $\Delta t_{n}=t_{n+1}-t_{n}$, and $\alpha, \beta \in[0,1]$ are parameters. It is well known that the scheme (2.6) is unconditionally stable for $\alpha, \beta \geq \frac{1}{2}$ and second-order accurate in time for $\alpha=\beta=\frac{1}{2}$.

The scheme (2.6) can be decoupled very easily. A well-known method in the engineering literature, the so-called Newmark method [13, 15], comes from (2.6) directly:

$$
\begin{aligned}
& B \xi_{n+1}=B \xi_{n}+\Delta t_{n} B \eta_{n}-\Delta t_{n}^{2}\left(\gamma A \xi_{n+1}+\left(\frac{1}{2}-\gamma\right) A \xi_{n}\right)+\Delta t_{n}^{2}\left(\gamma F_{n+1}+\left(\frac{1}{2}-\gamma\right) F_{n}\right), \\
& \text { (2.7b) } B \eta_{n+1}=B \eta_{n}-\Delta t_{n}\left(\alpha A \xi_{n+1}+(1-\alpha) A \xi_{n}\right)+\Delta t_{n}\left(\alpha F_{n+1}+(1-\alpha) F_{n}\right) .
\end{aligned}
$$

The Newmark method is unconditionally stable when $\alpha \geq \frac{1}{2}, \gamma \geq \frac{1}{4}$. Note that the matrix $B$ in (2.7) has condition number of size $O(1)$, and is diagonal when piecewise linear interpolation polynomials and appropriate integration quadrature rules [6] are applied. Thus, the introduction of the velocity $\eta$ as an independent variable does not significantly increase computational work. Indeed, the operation count is generally $O\left(m b^{2}\right)$ to solve for $\eta_{n+1}$ from (2.7b) when $\xi_{n+1}$ is known, where $m$ is the dimension of the space $S_{h}$ and $b$ the band width of the matrix $B$.

We note that, with $v=\frac{\partial u}{\partial t}$, the variational formulation (2.3) is equivalent to the following: find $u, v \in H_{0}^{1}(\Omega)$ such that for $t \in J$

$$
\left(\rho \frac{\partial v}{\partial t}, w\right)+(a \nabla u, \nabla w)=(f, w), \quad \forall w \in H_{0}^{1}(\Omega),
$$

$$
\left(\frac{\partial u}{\partial t}, w\right)=(v, w), \quad \forall w \in H_{0}^{1}(\Omega),
$$

$$
u(0)=u_{0}, \quad v(0)=v_{0} .
$$

The discrete schemes (2.6) and (2.7) may be thought of as being obtained directly from (2.8).

For the problem (2.1) and (2.2b), we have a similar variational formulation: find $u, v \in H^{1}(\Omega)$ satisfying

$$
\left(\rho \frac{\partial v}{\partial t}, w\right)+(a \nabla u, \nabla w)+\left\langle g+a_{1} u+a_{2} v, w\right\rangle=(f, w), \quad \forall w \in H^{1}(\Omega),
$$

$$
\begin{gathered}
\left(\frac{\partial u}{\partial t}, w\right)=(v, w), \quad \forall w \in H^{1}(\Omega), \\
u(0)=u_{0}, \quad v(0)=v_{0},
\end{gathered}
$$

where

$$
\langle f, g\rangle=\int_{\Gamma} f g d s
$$


Discrete schemes similar to (2.6) and (2.7) can be constructed from the variational form (2.9).

\section{NUMERICAL SCHEMES USING DYNAMIC FINITE ELEMENTS}

In this section we construct dynamic finite element schemes directly from the variational formulations $(2.8)$ and (2.9). We recall that the time interval $J=$ $(0, T]$ is partitioned as $0=t_{0}<t_{1}<\cdots<T_{N}=T$, and that $\Delta t_{n}=t_{n+1}-t_{n}$. For any function $w: \Omega \times J \rightarrow \mathbb{R}$, we denote $w_{n}=w\left(\cdot, t_{n}\right)$.

In order to accurately capture moving singularities such as traveling wave fronts, and let the size of the resulting linear systems be manageable, one needs to apply fine grids in regions near wave fronts, and relatively coarse grids in regions far away from wave fronts. As time goes on, the fine and coarse grid regions need to be modified or regenerated dynamically.

We first consider the problem (2.8). At time $t=t_{n}$, we let $T_{n}=\{K\}$ be a spatial discretization of the domain $\Omega$, and assume that the finite element space $S_{n} \subset H_{0}^{1}(\Omega)$ satisfies the following approximation property:

$$
\begin{aligned}
& \inf _{w_{h} \in S_{n}}\left\|w-w_{h}\right\|_{j} \leq C\left(\sum_{K \in T_{n}} h_{n, K}^{2(k+1-j)}\|w\|_{H^{k+1}(K)}^{2}\right)^{\frac{1}{2}}, \\
& j=0,1, \quad \forall w \in H^{k+1}(\Omega),
\end{aligned}
$$

where $h_{n, K}$ is the diameter of the element $K \in T_{n}$ and $k$ the order of the piecewise interpolation polynomials. The constant $C$ in (3.1) is assumed to be independent of $n$.

Introduce elliptic and $L^{2}$ projections of $u$ and $v:$ find $R_{n} u(\cdot, t), P_{n} v(\cdot, t) \in$ $S_{n}$ such that for any $t \in J$

$$
\begin{gathered}
\left(a \nabla\left(R_{n} u-u\right), \nabla w\right)=0, \quad \forall w \in S_{n}, \\
\left(\rho\left(P_{n} v-v\right), w\right)=0, \quad \forall w \in S_{n} .
\end{gathered}
$$

By (3.1) we have the following error bounds:

$$
\begin{aligned}
& \left\|R_{n} u-u\right\|_{j} \leq C\left(\sum_{K \in T_{n}} h_{n, K}^{2(k+1-j)}\|u\|_{H^{k+1}(K)}^{2}\right)^{\frac{1}{2}}, \quad j=0,1, \\
& \left\|P_{n} v-v\right\|_{j} \leq C\left(\sum_{K \in T_{n}} h_{n, K}^{2(k+1-j)}\|v\|_{H^{k+1}(K)}^{2}\right)^{\frac{1}{2}}, \quad j=0,1 .
\end{aligned}
$$

We are now in a position to define our numerical schemes for the problem (2.8), or equivalently, for (2.1) and (2.2a). Let $U_{0}, V_{0}$ be initial approximations of $u_{0}, v_{0}$, respectively; we define $U_{n+1}, V_{n+1} \in S_{n+1}$ to be the approximations of $u_{n+1}$ and $v_{n+1}$ satisfying

$$
\begin{gathered}
\left(\rho \frac{V_{n+1}-P_{n+1} V_{n}}{\Delta t_{n}}, w\right)+\left(a \nabla\left(\alpha U_{n+1}+(1-\alpha) R_{n+1} U_{n}\right), \nabla w\right) \\
=\left(\alpha f_{n+1}+(1-\alpha) f_{n}, w\right), \quad \forall w \in S_{n+1}, \\
\left(\frac{U_{n+1}-R_{n+1} U_{n}}{\Delta t_{n}}, w\right)=\left(\beta V_{n+1}+(1-\beta) P_{n+1} V_{n}, w\right), \quad \forall w \in S_{n+1},
\end{gathered}
$$


for $n=0,1, \ldots, N-1$; where $\alpha, \beta \in[0,1]$ are parameters.

In this numerical procedure, the solutions at the previous time level are first projected into the current finite element space, and then used as initial values to start a new time stepping. These projections are essential to get convergence of our numerical schemes. Note that the coefficient matrices resulting from computing the projections $P_{n+1} V_{n}$ and $R_{n+1} U_{n}$ can be utilized in the process of forming the stiffness matrix for the scheme (3.5). Thus, not much extra work is required to solve the scheme (3.5), compared with the scheme (2.6). Also note that when $S_{n}=S_{n+1}$ for all $n$, this procedure reduces to the standard scheme (2.6). It is easily seen that the procedure (3.5) permits a unique solution at each time step. Just as the Newmark method is obtained from (2.6), a modified Newmark method can be obtained from (3.5).

Theorem 3.1. The procedure (3.5) is unconditionally stable when $\alpha, \beta \geq \frac{1}{2}$.

Proof. From (3.5b) we see that

$$
\frac{U_{n+1}-R_{n+1} U_{n}}{\Delta t_{n}}=\beta V_{n+1}+(1-\beta) P_{n+1} V_{n} .
$$

Letting $w=\left(U_{n+1}-R_{n+1} U_{n}\right) / \Delta t_{n}$ in the second term on the left-hand side of (3.5a), and $w=\beta V_{n+1}+(1-\beta) P_{n+1} V_{n}$ for the other terms, we get

$$
\begin{aligned}
& \left(\rho \frac{V_{n+1}-P_{n+1} V_{n}}{\Delta t_{n}}, \beta V_{n+1}+(1-\beta) P_{n+1} V_{n}\right) \\
& \quad+\left(a \nabla\left(\alpha U_{n+1}+(1-\alpha) R_{n+1} U_{n}\right), \nabla \frac{U_{n+1}-R_{n+1} U_{n}}{\Delta t_{n}}\right) \\
& =\left(\alpha f_{n+1}+(1-\alpha) f_{n}, \beta V_{n+1}+(1-\beta) P_{n+1} V_{n}\right) .
\end{aligned}
$$

When $\alpha, \beta \geq \frac{1}{2}$, from (3.6) we easily see that

$$
\begin{gathered}
\frac{1}{2 \Delta t_{n}}\left[\left\|V_{n+1}\right\|_{\rho}^{2}-\left\|P_{n+1} V_{n}\right\|_{\rho}^{2}+\left\|\nabla U_{n+1}\right\|_{a}^{2}-\left\|\nabla R_{n+1} U_{n}\right\|_{a}^{2}\right] \\
\leq C\left[\left\|f_{n+1}\right\|^{2}+\left\|f_{n}\right\|^{2}+\left\|V_{n+1}\right\|_{\rho}^{2}+\left\|P_{n+1} V_{n}\right\|_{\rho}^{2}\right] .
\end{gathered}
$$

By the definition of the projections $P_{n}$ and $R_{n}$, (3.7) implies

$$
\begin{aligned}
& \left\|V_{n+1}\right\|_{\rho}^{2}-\left\|V_{n}\right\|_{\rho}^{2}+\left\|\nabla U_{n+1}\right\|_{a}^{2}-\left\|\nabla U_{n}\right\|_{a}^{2} \\
& \quad \leq C \Delta t_{n}\left[\left\|f_{n+1}\right\|^{2}+\left\|f_{n}\right\|^{2}+\left\|V_{n+1}\right\|_{\rho}^{2}+\left\|V_{n}\right\|_{\rho}^{2}\right] .
\end{aligned}
$$

An application of Gronwall's lemma to (3.8) yields

$$
\left\|V_{n}\right\|_{\rho}^{2}+\left\|\nabla U_{n}\right\|_{a}^{2} \leq\left\|V_{0}\right\|_{\rho}^{2}+\left\|\nabla U_{0}\right\|_{a}^{2}+C \max _{0 \leq i \leq n-1}\left\{\Delta t_{i}\right\} \sum_{i=0}^{n}\left\|f_{i}\right\|^{2} .
$$

When $f \equiv 0$, we have

$$
\left\|V_{n}\right\|_{\rho}^{2}+\left\|\nabla U_{n}\right\|_{\rho}^{2} \leq\left\|V_{0}\right\|_{\rho}^{2}+\left\|\nabla U_{0}\right\|_{a}^{2}, \quad \text { for } n=1,2, \cdots, N .
$$

This completes the proof.

Now consider the variational formulation (2.9) for the problem (2.1) and (2.2b). At time $t=t_{n}$, let $S_{n} \subset H^{1}(\Omega)$ be a finite element space satisfying 
(3.1). Define the operator $P_{n}$ by (3.3), and $R_{n}$ by the following, instead of (3.2),

$$
\left(a \nabla\left(R_{n} u-u\right), \nabla w\right)+\left\langle a_{1}\left(R_{n} u-u\right), w\right\rangle=0, \quad \forall w \in S_{n} .
$$

Note that the standard coercivity property does not hold for the problem (3.11). When $a_{1}=0$ a.e. on $\Gamma$, the existence and uniqueness of the solution to equation (3.11) and error bounds (3.4) can be derived by seeking a solution $R_{n} u$ in the quotient space $S_{n} /\{$ constants $\}$, or $S_{n} /\left\{w \mid \int_{\Omega} w d x=0\right\}$, and applying Poincaré's inequality; otherwise, by applying the duality argument of Schatz [5, 19,20 ] and Garding's inequality, we still can derive the error bounds (3.4), under some assumptions on the regularity of the functions $a, a_{1}$ and the boundary $\Gamma$.

Again, let $U_{0}, V_{0}$ be initial approximations of $u_{0}, v_{0}$, respectively. We define $U_{n+1}, V_{n+1} \in S_{n+1}$ to be the approximations of $u_{n+1}$ and $v_{n+1}$ such that for $n=0,1, \ldots, N-1$,

$$
\begin{aligned}
&\left(\rho \frac{V_{n+1}-P_{n+1} V_{n}}{\Delta t_{n}}, w\right)+\left(a \nabla\left(\alpha U_{n+1}+(1-\alpha) R_{n+1} U_{n}\right), \nabla w\right) \\
&+\left\langle\alpha g_{n+1}+(1-\alpha) g_{n}+a_{1}\left(\alpha U_{n+1}\right.\right.\left.+(1-\alpha) R_{n+1} U_{n}\right) \\
&\left.+a_{2}\left(\alpha V_{n+1}+(1-\alpha) P_{n+1} V_{n}\right), w\right\rangle \\
&=\left(\alpha f_{n+1}+(1-\alpha) f_{n}, w\right), \quad \forall w \in S_{n+1},
\end{aligned}
$$

$$
\left(\frac{U_{n+1}-R_{n+1} U_{n}}{\Delta t_{n}}, w\right)=\left(\alpha V_{n+1}+(1-\alpha) P_{n+1} V_{n}, w\right), \quad \forall w \in S_{n+1},
$$

where $\alpha \in[0,1]$ is a parameter.

By a similar argument to that used in the proof of Theorem 3.1, we have the following stability result.

Theorem 3.2. The procedure (3.12) is unconditionally stable when $\alpha \in\left[\frac{1}{2}, 1\right]$.

\section{ERROR ESTIMATES}

In this section we assume that $\alpha, \beta \geq \frac{1}{2}$ in the schemes (3.5) and (3.12), and adopt the following notation:

$$
\begin{array}{ll}
e_{n}=U_{n}-R_{n} u_{n}, & \bar{e}_{n}=R_{n+1} U_{n}-R_{n+1} u_{n}, \\
r_{n}=V_{n}-P_{n} v_{n}, & \hat{r}_{n}=P_{n+1} V_{n}-P_{n+1} v_{n}, \\
\epsilon_{n}=u_{n}-R_{n} u_{n}, & \bar{\epsilon}_{n}=u_{n}-R_{n+1} u_{n}, \\
\delta_{n}=v_{n}-P_{n} v_{n}, & \hat{\delta}_{n}=v_{n}-P_{n+1} v_{n},
\end{array}
$$

and for simplicity choose the initial values $\left\{U_{0}, V_{0}\right\}$ such that $e_{0}=r_{0}=0$.

First consider the scheme (3.5). Note that $(2.8 \mathrm{a})$ can be rewritten as

$$
\begin{aligned}
& \left(\rho\left(\alpha \frac{\partial v_{n+1}}{\partial t}+(1-\alpha) \frac{\partial v_{n}}{\partial t}\right), w\right)+\left(a \nabla\left(\alpha u_{n+1}+(1-\alpha) u_{n}\right), \nabla w\right) \\
& \quad=\left(\alpha f_{n+1}+(1-\alpha) f_{n}, w\right), \quad \forall w \in H_{0}^{1}(\Omega) .
\end{aligned}
$$


By (3.2) we can rewrite (4.1) as

$$
\begin{aligned}
& \left(\rho\left(\alpha \frac{\partial v_{n+1}}{\partial t}+(1-\alpha) \frac{\partial v_{n}}{\partial t}\right), w\right)+\left(a \nabla\left(\alpha R_{n+1} u_{n+1}+(1-\alpha) R_{n+1} u_{n}\right), \nabla w\right) \\
& \quad=\left(\alpha f_{n+1}+(1-\alpha) f_{n}, w\right), \quad \forall w \in S_{n+1} .
\end{aligned}
$$

Combining (4.2), (3.5a) and (3.3), we have

$$
\begin{aligned}
& \left(\rho \frac{r_{n+1}-\hat{r}_{n}}{\Delta t_{n}}, w\right)+\left(a \nabla\left(\alpha e_{n+1}+(1-\alpha) \bar{e}_{n}\right), \nabla w\right) \\
& \quad=\left(\rho\left(\alpha \frac{\partial v_{n+1}}{\partial t}+(1-\alpha) \frac{\partial v_{n}}{\partial t}\right)-\rho \frac{v_{n+1}-v_{n}}{\Delta t_{n}}, w\right), \quad \forall w \in S_{n+1} .
\end{aligned}
$$

By (3.5b) we have

$$
\begin{aligned}
& \frac{e_{n+1}-\bar{e}_{n}}{\Delta t_{n}}+\frac{R_{n+1} u_{n+1}-R_{n+1} u_{n}}{\Delta t_{n}} \\
& \quad=\beta r_{n+1}+(1-\beta) \hat{r}_{n}+\beta P_{n+1} v_{n+1}+(1-\beta) P_{n+1} v_{n} .
\end{aligned}
$$

Let $w=\beta r_{n+1}+(1-\beta) \hat{r}_{n}$ in (4.3) except for the second term on the left-hand side, where we let

$$
w=\frac{e_{n+1}-\bar{e}_{n}}{\Delta t_{n}}+\frac{R_{n+1} u_{n+1}-R_{n+1} u_{n}}{\Delta t_{n}}-\left(\beta P_{n+1} v_{n+1}+(1-\beta) P_{n+1} v_{n}\right) ;
$$

by (4.4), this is correct. Then we get the following error equation:

$$
\begin{gathered}
\left(\rho \frac{r_{n+1}-\hat{r}_{n}}{\Delta t_{n}}, \beta r_{n+1}+(1-\beta) \hat{r}_{n}\right)+\left(a \nabla\left(\alpha e_{n+1}+(1-\alpha) \bar{e}_{n}\right), \nabla \frac{e_{n+1}-\bar{e}_{n}}{\Delta t_{n}}\right) \\
=\left(\rho\left(\alpha \frac{\partial v_{n+1}}{\partial t}+(1-\alpha) \frac{\partial v_{n}}{\partial t}\right)-\rho \frac{v_{n+1}-v_{n}}{\Delta t_{n}}, \beta r_{n+1}+(1-\beta) \hat{r}_{n}\right) \\
-\left(a \nabla\left(\alpha e_{n+1}+(1-\alpha) \bar{e}_{n}\right),\right. \\
\left.\quad \nabla\left(\frac{R_{n+1} u_{n+1}-R_{n+1} u_{n}}{\Delta t_{n}}-\left(\beta P_{n+1} v_{n+1}+(1-\beta) P_{n+1} v_{n}\right)\right)\right) \\
\equiv T_{1}+T_{2} .
\end{gathered}
$$

Since $\alpha, \beta \geq \frac{1}{2}$, we easily see that

$$
\begin{aligned}
& \left(\rho \frac{r_{n+1}-\hat{r}_{n}}{\Delta t_{n}}, \beta r_{n+1}+(1-\beta) \hat{r}_{n}\right) \\
& =\frac{1}{2 \Delta t_{n}}\left[\left(\rho r_{n+1}, r_{n+1}\right)-\left(\rho \hat{r}_{n}, \hat{r}_{n}\right)\right] \\
& \quad+\frac{1}{\Delta t_{n}}\left[\left(\beta-\frac{1}{2}\right)\left(\rho r_{n+1}, r_{n+1}\right)+(1-2 \beta)\left(\rho r_{n+1}, \hat{r}_{n}\right)+\left(\beta-\frac{1}{2}\right)\left(\rho \hat{r}_{n}, \hat{r}_{n}\right)\right] \\
& \geq \frac{1}{2 \Delta t_{n}}\left[\left\|r_{n+1}\right\|_{\rho}^{2}-\left\|\hat{r}_{n}\right\|_{\rho}^{2}\right],
\end{aligned}
$$

and

$$
\left(a \nabla\left(\alpha e_{n+1}+(1-\alpha) \bar{e}_{n}\right), \nabla \frac{e_{n+1}-\bar{e}_{n}}{\Delta t_{n}}\right) \geq \frac{1}{2 \Delta t_{n}}\left[\left\|\nabla e_{n+1}\right\|_{a}^{2}-\left\|\nabla \bar{e}_{n}\right\|_{a}^{2}\right]
$$


Thus, the terms on the left-hand side of (4.5) dominate

$$
\frac{1}{2 \Delta t_{n}}\left[\left\|r_{n+1}\right\|_{\rho}^{2}-\left\|\hat{r}_{n}\right\|_{\rho}^{2}+\left\|\nabla e_{n+1}\right\|_{a}^{2}-\left\|\nabla \bar{e}_{n}\right\|_{a}^{2}\right] \text {. }
$$

On the other hand, for the terms on the right-hand side we have

$$
\left|T_{1}\right| \leq C\left[\left\|r_{n+1}\right\|_{\rho}^{2}+\left\|\hat{r}_{n}\right\|_{\rho}^{2}+\Delta t_{n}^{3} \int_{t_{n}}^{t_{n+1}}\left\|\frac{\partial^{2} v}{\partial t^{2}}\right\|^{2} d \tau+\Delta t_{n} \int_{t_{n}}^{t_{n+1}}\left\|\frac{\partial v}{\partial t}\right\|^{2} d \tau\right]
$$

$$
\begin{aligned}
& \left|T_{2}\right|=\mid\left(a \nabla\left(\alpha e_{n+1}+(1-\alpha) \bar{e}_{n}\right),\right. \\
& \left.\nabla\left[\frac{\epsilon_{n+1}-\bar{\epsilon}_{n}}{\Delta t_{n}}-\left(\beta \delta_{n+1}+(1-\beta) \hat{\delta}_{n}\right)+\left(-\frac{u_{n+1}-u_{n}}{\Delta t_{n}}+\beta v_{n+1}+(1-\beta) v_{n}\right)\right]\right) \mid \\
& \leq C\left[\left\|\nabla e_{n+1}\right\|_{a}^{2}+\left\|\nabla \bar{e}_{n}\right\|_{a}^{2}+\frac{1}{\Delta t_{n}^{2}}\left\|\nabla\left(\epsilon_{n+1}-\bar{\epsilon}_{n}\right)\right\|_{a}^{2}+\left\|\nabla \delta_{n+1}\right\|^{2}\right. \\
& \left.\quad+\left\|\nabla \hat{\delta}_{n}\right\|^{2}+\Delta t_{n}^{3} \int_{t_{n}}^{t_{n+1}}\left\|\nabla \frac{\partial^{2} u}{\partial t^{2}}\right\|^{2} d \tau+\Delta t_{n} \int_{t_{n}}^{t_{n+1}}\left\|\nabla \frac{\partial v}{\partial t}\right\|^{2} d \tau\right] .
\end{aligned}
$$

Combining (4.5)-(4.8), we have the following error inequality:

$$
\begin{aligned}
& \left\|r_{n+1}\right\|_{\rho}^{2}-\left\|\hat{r}_{n}\right\|_{\rho}^{2}+\left\|\nabla e_{n+1}\right\|_{a}^{2}-\left\|\nabla \bar{e}_{n}\right\|_{a}^{2} \\
& \leq C\left[\Delta t_{n}\left(\left\|r_{n+1}\right\|_{\rho}^{2}+\left\|\hat{r}_{n}\right\|_{\rho}^{2}+\left\|\nabla e_{n+1}\right\|_{a}^{2}+\left\|\nabla \bar{e}_{n}\right\|_{a}^{2}\right)+E_{n}\right],
\end{aligned}
$$

where

$$
\begin{aligned}
E_{n}= & \frac{1}{\Delta t_{n}}\left\|\epsilon_{n+1}-\bar{\epsilon}_{n}\right\|_{1}^{2}+\Delta t_{n}\left(\left\|\delta_{n+1}\right\|_{1}^{2}+\left\|\hat{\delta}_{n}\right\|_{1}^{2}\right) \\
& +\Delta t_{n}^{2} \int_{t_{n}}^{t_{n+1}}\left(\left\|\frac{\partial^{3} u}{\partial t^{3}}\right\|^{2}+\left\|\nabla \frac{\partial^{2} u}{\partial t^{2}}\right\|^{2}+\left\|\frac{\partial^{2} u}{\partial t^{2}}\right\|^{2}\right) d \tau .
\end{aligned}
$$

Now by the definition of the operator $R_{n+1}$,

$$
\left(a \nabla\left(R_{n+1} U_{n}-U_{n}\right), \nabla w\right)=0, \quad \forall w \in S_{n+1},
$$

we have

$$
\left(a \nabla\left(\bar{e}_{n}-e_{n}\right), \nabla w\right)=\left(a \nabla\left(\bar{\epsilon}_{n}-\epsilon_{n}\right), \nabla w\right), \quad \forall w \in S_{n+1} .
$$

Setting $w=\bar{e}_{n}$ and applying the inequality $a b \leq \frac{1}{2}\left(a^{2} / \epsilon+\epsilon b^{2}\right)$ with $\epsilon=1-\xi$ to the term on the right-hand side, we obtain

$$
\xi\left\|\nabla \bar{e}_{n}\right\|_{a}^{2}-\left\|\nabla e_{n}\right\|_{a}^{2} \leq \frac{1}{1-\xi}\left\|\nabla\left(\bar{\epsilon}_{n}-\epsilon_{n}\right)\right\|_{a}^{2}, \quad \forall \xi \in(0,1) .
$$

Similarly, from the definition of the operator $P_{n+1}$,

$$
\left(\rho\left(P_{n+1} V_{n}-V_{n}\right), w\right)=0, \quad \forall w \in S_{n+1},
$$

we see that

$$
\xi\left\|\hat{r}_{n}\right\|_{\rho}^{2}-\left\|r_{n}\right\|_{\rho}^{2} \leq \frac{1}{1-\xi}\left\|\hat{\delta}_{n}-\delta_{n}\right\|_{\rho}^{2}, \quad \forall \xi \in(0,1) .
$$


Combining (4.9), (4.11) and (4.12) yields

$$
\begin{aligned}
& \xi\left\|r_{n+1}\right\|_{\rho}^{2}-\left\|r_{n}\right\|_{\rho}^{2}+\xi\left\|\nabla e_{n+1}\right\|_{a}^{2}-\left\|\nabla e_{n}\right\|_{a}^{2} \\
& \leq C \\
& \quad\left[\Delta t_{n}\left(\left\|r_{n+1}\right\|_{\rho}^{2}+\left\|r_{n}\right\|_{\rho}^{2}+\left\|\nabla e_{n+1}\right\|_{a}^{2}+\left\|\nabla e_{n}\right\|_{a}^{2}\right)\right. \\
& \left.\quad+E_{n}+\frac{1}{1-\xi}\left(\left\|\bar{\epsilon}_{n}-\epsilon_{n}\right\|_{1}^{2}+\left\|\hat{\delta}_{n}-\delta_{n}\right\|^{2}\right)\right], \quad \forall \xi \in(0,1) .
\end{aligned}
$$

This is the error relation in case of implementing different grids at different times. However, from the computational point of view, we may want to choose some larger fine-grid areas to capture wave fronts for several time steps, and change the grid after several time steps only. When the grid is the same at times $t=t_{n}$ and $t=t_{n+1}$, then the error relation becomes

$$
\begin{aligned}
& \left\|r_{n+1}\right\|_{\rho}^{2}-\left\|r_{n}\right\|_{\rho}^{2}+\left\|\nabla e_{n+1}\right\|_{a}^{2}-\left\|\nabla e_{n}\right\|_{a}^{2} \\
& \leq C\left[\Delta t_{n}\left(\left\|r_{n+1}\right\|_{\rho}^{2}+\left\|r_{n}\right\|_{\rho}^{2}+\left\|\nabla e_{n+1}\right\|_{a}^{2}+\left\|\nabla e_{n}\right\|_{a}^{2}\right)+E_{n}\right] .
\end{aligned}
$$

Let $M_{m}$ be the number of changes of spatial discretizations from time $t=$ $t_{0}$ to time $t=t_{m}$. From the error relations (4.13) and (4.14), applying the argument given in [24], we obtain for $1 \leq m \leq N$

$$
\begin{aligned}
\left\|r_{m}\right\|_{\rho}^{2}+\left\|\nabla e_{m}\right\|_{a}^{2} \leq C & {\left[\sum_{n=0}^{m-1} \Delta t_{n}\left(\left\|r_{n+1}\right\|_{\rho}^{2}+\left\|r_{n}\right\|_{\rho}^{2}+\left\|\nabla e_{n+1}\right\|_{a}^{2}+\left\|\nabla e_{n}\right\|_{a}^{2}\right)\right.} \\
& \left.+\sum_{n=0}^{m-1} E_{n}+M_{m} \sum_{n=0}^{m-1}\left(\left\|\bar{\epsilon}_{n}-\epsilon_{n}\right\|_{1}^{2}+\left\|\bar{\delta}_{n}-\delta_{n}\right\|^{2}\right)\right] .
\end{aligned}
$$

By Gronwall's lemma we have

$$
\left\|r_{m}\right\|_{\rho}^{2}+\left\|\nabla e_{m}\right\|_{a}^{2} \leq C\left[\sum_{n=0}^{m-1} E_{n}+M_{m}^{2} \cdot \max _{0 \leq n \leq m-1}\left\{\left\|\bar{\epsilon}_{n}-\epsilon_{n}\right\|_{1}^{2}+\left\|\bar{\delta}_{n}-\delta_{n}\right\|^{2}\right\}\right] .
$$

Note that the straightforward bound of the first term in the definition of $E_{n}$ would introduce a factor $O\left(\frac{1}{\Delta t}\right)$ into our error estimates. Thus, we adopt the following procedure:

$$
\begin{aligned}
\left\|\epsilon_{n+1}-\bar{\epsilon}_{n}\right\|_{1}^{2} & =\left\|\left(I-R_{n+1}\right)\left(u_{n+1}-u_{n}\right)\right\|_{1}^{2} \\
& \leq C\left[\sum_{K \in T_{n+1}} h_{n+1, K}^{2 k}\left\|u_{n+1}-u_{n}\right\|_{H^{k+1}(K)}^{2}\right] \\
& =C\left[\sum_{K \in T_{n+1}} h_{n+1, K}^{2 k}\left\|\int_{t_{n}}^{t_{n+1}} \frac{\partial u}{\partial t}(\tau) d \tau\right\|_{H^{k+1}(K)}^{2}\right] \\
& \leq C \Delta t_{n} \sum_{K \in T_{n+1}} h_{n+1, K}^{2 k} \int_{t_{n}}^{t_{n+1}}\left\|u_{t}\right\|_{H^{k+1}(K)}^{2} d \tau .
\end{aligned}
$$


By (4.16), (4.17), (4.10), (3.4) and the triangular inequality we immediately have the following theorem.

Theorem 4.1. Let $u$ be the solution of (2.1) and (2.2a), and $v=\frac{\partial u}{\partial t}$. Let $U$ and $V$ be the solution of the scheme (3.5) with $\alpha, \beta \geq \frac{1}{2}$. Then, for sufficiently small $\Delta t_{n}(0 \leq n \leq N-1)$, we have the error bounds for $m=1,2, \ldots, N$, (4.18)

$$
\begin{aligned}
\| V_{m} & -v_{m}\left\|^{2}+\right\| U_{m}-u_{m} \|_{1}^{2} \\
\leq & C\left\{\sum_{n=0}^{m-1} \int_{t_{n}}^{t_{n+1}} \sum_{K \in T_{n+1}} h_{n+1, K}^{2 k}\left\|u_{t}\right\|_{H^{k+1}(K)}^{2} d \tau\right. \\
& +\sum_{n=0}^{m-1} \Delta t_{n}^{2} \int_{t_{n}}^{t_{n+1}}\left(\left\|u_{t t t}\right\|^{2}+\left\|u_{t t}\right\|^{2}+\left\|\nabla u_{t t}\right\|^{2}\right) d \tau \\
& +\max _{n \leq m-1}\left[\sum_{K \in T_{n+1}} h_{n+1, K}^{2 k}\left(\left\|\frac{\partial u_{n}}{\partial t}\right\|_{H^{k+1}(K)}^{2}+\left\|\frac{\partial u_{n+1}}{\partial t}\right\|_{H^{k+1}(K)}^{2}\right)\right] \\
& +M_{m}^{2} \cdot \max _{n \leq m-1}\left[\sum_{K \in T_{n+1}} h_{n+1, K}^{2 k}\left(\left\|u_{n}\right\|_{H^{k+1}(K)}^{2}+\left\|\frac{\partial u_{n}}{\partial t}\right\|_{H^{k+1}(K)}^{2}\right)\right. \\
& \left.\left.+\sum_{K \in T_{n}} h_{n, K}^{2 k}\left(\left\|u_{n}\right\|_{H^{k+1}(K)}^{2}+\left\|\frac{\partial u_{n}}{\partial t}\right\|_{H^{k+1}(K)}^{2}\right)\right]+\sum_{K \in T_{m}} h_{m, K}^{2 k}\left\|u_{m}\right\|_{H^{k+1}(K)}^{2}\right\},
\end{aligned}
$$

where $M_{m}$ is the number of different finite element discretizations from $t=t_{0}$ to $t=t_{m}$.

We now estimate the error for the scheme (3.12). Combining (3.12a), (2.9), (3.2) and (3.3), we have

$$
\begin{aligned}
& \left(\rho \frac{r_{n+1}-\hat{r}_{n}}{\Delta t_{n}}, w\right)+\left(a \nabla\left(\alpha e_{n+1}+(1-\alpha) \bar{e}_{n}\right), \nabla w\right) \\
& \quad+\left\langle a_{1}\left(\alpha e_{n+1}+(1-\alpha) \bar{e}_{n}\right)+a_{2}\left(\alpha r_{n+1}+(1-\alpha) \hat{r}_{n}\right), w\right\rangle \\
& =\left(\rho\left(\alpha \frac{\partial v_{n+1}}{\partial t}+(1-\alpha) \frac{\partial v_{n}}{\partial t}\right)-\rho \frac{v_{n+1}-v_{n}}{\Delta t_{n}}, w\right) \\
& \quad+\left\langle a_{2}\left(\alpha \delta_{n+1}+(1-\alpha) \hat{\delta}_{n}\right), w\right\rangle, \quad \forall w \in S_{n+1} .
\end{aligned}
$$

The equation (4.19) is derived just like (4.3). Note that $(3.12 \mathrm{~b})$ is equivalent to the following equation:

$$
\begin{aligned}
& \frac{e_{n+1}-\bar{e}_{n}}{\Delta t_{n}}+\frac{R_{n+1} u_{n+1}-R_{n+1} u_{n}}{\Delta t_{n}} \\
& =\alpha r_{n+1}+(1-\alpha) \hat{r}_{n}+\alpha P_{n+1} v_{n+1}+(1-\alpha) P_{n+1} v_{n} .
\end{aligned}
$$

Letting $w=\alpha r_{n+1}+(1-\alpha) \hat{r}_{n}$ in (4.19) and making use of (4.20), we obtain 
the error equation

(4.21)

$$
\begin{aligned}
& \left(\rho \frac{r_{n+1}-\hat{r}_{n}}{\Delta t_{n}}, \alpha r_{n+1}+(1-\alpha) \hat{r}_{n}\right)+\left(a \nabla\left(\alpha e_{n+1}+(1-\alpha) \bar{e}_{n}\right), \nabla \frac{e_{n+1}-\bar{e}_{n}}{\Delta t_{n}}\right) \\
& \quad+\left\langle a_{1}\left(\alpha e_{n+1}+(1-\alpha) \bar{e}_{n}\right), \frac{e_{n+1}-\bar{e}_{n}}{\Delta t_{n}}\right\rangle \\
& \quad+\left\langle a_{2}\left(\alpha r_{n+1}+(1-\alpha) \hat{r}_{n}\right), \alpha r_{n+1}+(1-\alpha) \hat{r}_{n}\right\rangle \\
& =\left(\rho\left(\alpha \frac{\partial v_{n+1}}{\partial t}+(1-\alpha) \frac{\partial v_{n}}{\partial t}\right)-\rho \frac{v_{n+1}-v_{n}}{\Delta t_{n}}, \alpha r_{n+1}+(1-\alpha) \hat{r}_{n}\right) \\
& \quad-\left(a \nabla\left(\alpha e_{n+1}+(1-\alpha) \bar{e}_{n}\right),\right. \\
& \left.\quad \nabla\left(\frac{R_{n+1} u_{n+1}-R_{n+1} u_{n}}{\Delta t_{n}}-\left(\alpha P_{n+1} v_{n+1}+(1-\alpha) P_{n+1} v_{n}\right)\right)\right) \\
& -\left\langle a_{1}\left(\alpha e_{n+1}+(1-\alpha) \bar{e}_{n}\right), \frac{R_{n+1} u_{n+1}-R_{n+1} u_{n}}{\Delta t_{n}}-\left(\alpha P_{n+1} v_{n+1}+(1-\alpha) P_{n+1} v_{n}\right)\right\rangle \\
& +\left\langle a_{2}\left(\alpha \delta_{n+1}+(1-\alpha) \hat{\delta}_{n}\right), \alpha r_{n+1}+(1-\alpha) \hat{r}_{n}\right\rangle .
\end{aligned}
$$

Using (4.21), the fact that $a_{1}, a_{2}$ are multipliers on $H^{\frac{1}{2}}(\Gamma)$ and the trace theorem, and applying the argument used in the derivation of (4.9), we have the error inequality

$$
\begin{aligned}
& \left\|r_{n+1}\right\|_{\rho}^{2}-\left\|\hat{r}_{n}\right\|_{\rho}^{2}+\left\|\nabla e_{n+1}\right\|_{a}^{2}-\left\|\nabla \bar{e}_{n}\right\|_{a}^{2}+\left|e_{n+1}\right|_{a_{1}}^{2}-\left|\bar{e}_{n}\right|_{a_{1}}^{2} \\
& \leq C\left[\Delta t_{n}\left(\left\|r_{n+1}\right\|_{\rho}^{2}+\left\|\hat{r}_{n}\right\|_{\rho}^{2}+\left\|\nabla e_{n+1}\right\|_{a}^{2}+\left\|\nabla \bar{e}_{n}\right\|_{a}^{2}+\left|e_{n+1}\right|_{a_{1}}^{2}+\left|\bar{e}_{n}\right|_{a_{1}}^{2}\right)+E_{n}\right],
\end{aligned}
$$

where $E_{n}$ is defined by $(4.10)$.

Recall that the projection $R_{n}$ is defined by (3.11). Similarly to (4.11), we have

$$
\begin{aligned}
& \xi\left\|\nabla \bar{e}_{n}\right\|_{a}^{2}-\left\|\nabla e_{n}\right\|_{a}^{2}+\xi\left|\bar{e}_{n}\right|_{a_{1}}^{2}-\left|e_{n}\right|_{a_{1}}^{2} \\
& \quad \leq \frac{1}{1-\xi}\left[\left\|\nabla\left(\bar{\epsilon}_{n}-\epsilon_{n}\right)\right\|_{a}^{2}+\left|\bar{\epsilon}_{n}-\epsilon_{n}\right|_{a_{1}}^{2}\right], \quad \forall \xi \in(0,1) .
\end{aligned}
$$

From (4.22), (4.23) and (4.12) we obtain

$$
\begin{aligned}
\left\|r_{m}\right\|_{\rho}^{2}+ & \left\|\nabla e_{m}\right\|_{a}^{2}+\left|e_{m}\right|_{a_{1}}^{2} \\
\leq C & {\left[\sum_{n=0}^{m-1} \Delta t_{n}\left(\left\|r_{n+1}\right\|_{\rho}^{2}+\left\|r_{n}\right\|_{\rho}^{2}+\left\|\nabla e_{n+1}\right\|_{a}^{2}+\left\|\nabla e_{n}\right\|_{a}^{2}+\left|e_{n+1}\right|_{a_{1}}^{2}+\left|e_{n}\right|_{a_{1}}^{2}\right)\right.} \\
& \left.+\sum_{n=0}^{m-1} E_{n}+M_{m} \sum_{n=0}^{m-1}\left(\left\|\nabla\left(\bar{\epsilon}_{n}-\epsilon_{n}\right)\right\|^{2}+\left|\bar{\epsilon}_{n}-\epsilon_{n}\right|_{a_{1}}^{2}+\left\|\bar{\delta}_{n}-\delta_{n}\right\|^{2}\right)\right] .
\end{aligned}
$$

The error relation (4.24) is derived just like (4.15). Now an application of Gronwall's lemma, the trace inequality and the triangular inequality leads to the following theorem.

Theorem 4.2. Let $u$ be the solution of (2.1) and (2.2b), and $v=\frac{\partial u}{\partial t}$. Let $U$ and $V$ be the solution of the scheme (3.12) with $\alpha \geq \frac{1}{2}$. Then, for sufficiently 
small $\Delta t_{n}(0 \leq n \leq N-1)$, we have the error bounds

(4.25)

$$
\left\|V_{m}-v_{m}\right\|^{2}+\left\|\nabla\left(U_{m}-u_{m}\right)\right\|^{2} \leq[\text { the right-hand side terms of }(4.18)] \text {. }
$$

\section{REMARKS}

We have presented a family of grid modification finite element schemes and derived error estimates for them. In this section we make a few remarks.

Remark 5.1. The schemes (3.5) and (3.12) can easily include the case in which the interpolation polynomials are adjusted dynamically [24]. In particular, the $h p$-version of the finite element methods [1] can be applied at each time step.

Remark 5.2. The scheme (3.5) has order $O\left(\Delta t^{2}\right)$ when $\alpha=\beta=\frac{1}{2}$. This can be easily proved by modifying the proof of Theorem 4.1. Similarly, the scheme (3.12) is of order $O\left(\Delta t^{2}\right)$ when $\alpha=\frac{1}{2}$.

Remark 5.3. The error estimates in Theorems 4.1 and 4.2 are optimal in the energy norm sense when the total number of different finite element discretizations is bounded. However, they are one order from being optimal in the $L^{2}$ norm sense.

Remark 5.4. A priori error estimates similar to (4.18) and (4.25) were used in $[15, \S 8.4 .4$ and $\S 4.6]$ to design adaptive algorithms for grid modification and time step control. A numerical example based on a posteriori use of a priori estimates can be found in [15, Example 9.6].

Remark 5.5. Our error estimates in Theorems 4.1 and 4.2 reduce to the standard ones when no grid modification is made and a quasi-uniform grid is adopted.

Remark 5.6. The error estimates in Theorems 4.1 and 4.2 imply that frequent changes of the finite element discretization may have some influence on the accuracy of the method. Thus, in practical computations, we might want to choose larger fine-grid regions to track wave fronts for several time steps, and modify the grid after several time steps only.

Remark 5.7. For nonlinear problems, in order to avoid nonlinearity of the resulting systems of algebraic equations, second-order schemes such as predictorcorrector schemes and extrapolated Crank-Nicolson schemes [22, 23] can be constructed. Error estimates follow analogously.

Remark 5.8. When the problem (2.1) has an absorbing boundary condition

$$
a \frac{\partial u}{\partial \nu}+a_{2} \frac{\partial u}{\partial t}=0, \quad(x, t) \in \Gamma \times J,
$$

we may also introduce the weighted elliptic projection $R_{n}$ as

$$
\left(a \nabla\left(R_{n} u-u\right), \nabla w\right)+\left(R_{n} u-u, w\right)=0, \quad \forall w \in S_{n},
$$

in place of (3.11). This way, we can avoid the duality argument of Schatz and get the error bounds (3.4) directly. Besides, the error estimates for the scheme (3.12) will have the form (4.18) instead of (4.25). Note that the problem (5.2) has more stability than the problem (3.11). Thus, whenever the boundary condition (5.1) is given for the problem (2.1), the projection (5.2) should be used. 
Remark 5.9. Wave fronts are usually easier to capture than other critical features, e.g., moving fluid interfaces in reservoir simulation. Note that the wave speed can be directly derived from the coefficients in the equation (2.1a). If we know where the wave fronts are at a given time, by the wave speed and the direction of propagation, we can easily tell where the wave fronts will be at the next time.

Remark 5.10. Grid modification can be incorporated with domain decomposition methods for second-order hyperbolic problems as easily as for parabolic problems [25]. Dynamic domain decompositions can be made according to the changing nature of the exact solution. Then uniform fine grids or local grid refinements can be applied in subdomains which contain local critical features, and uniform coarse grids or local grid coarsenings can be applied in other subdomains. Theoretical analysis and numerical experiments [25] show that this technique leads to an effective way for capturing moving local phenomena. When parallel computers are used, this technique can also provide a mechanism for load balancing.

\section{ACKNOWLEDGMENT}

The author would like to thank Professor Jim Douglas, Jr. and Professor Juan E. Santos for their encouragement.

\section{BIBLIOGRAPHY}

1. I. Babuška and M. Suri, p-and hp-versions of the FEM, an overview, Comput. Methods Appl. Mech. Engrg. 80 (1990), 5-26.

2. M.J. Baines, An analysis of the moving finite element procedure, SIAM J. Numer. Anal. 28 (1991), 1323-1349.

3. G.A. Baker, Error estimates for finite element methods for second order hyperbolic equations, SIAM J. Numer. Anal. 13 (1976), 564-576.

4. R.E. Bank and R.F. Santos, Analysis of some moving space-time finite element methods, SIAM J. Numer. Anal. 30 (1993), 1-18.

5. J.H. Bramble and J.E. Osborn, Rate of convergence estimates for nonselfadjoint eigenvalue approximations, Math. Comp. 27 (1973), 525-549.

6. P.G. Ciarlet, The finite element method for elliptic problems, North-Holland, Amsterdam, 1978.

7. R. Clayton and B. Engquist, Absorbing boundary condition for acoustic and elastic wave equations, Bull. Seismol. Soc. Amer. 67 (1977), 1529-1540.

8. L.C. Cowsar, T.F. Dupont, and M.F. Wheeler, A priori estimates for mixed finite element methods for the wave equation, Comput. Methods Appl. Mech. Engrg. 82 (1990), 205-222.

9. J. Douglas, Jr. and T.F. Dupont, Galerkin methods for parabolic equations with nonlinear boundary conditions, Numer. Math. 20 (1973), 213-237.

10. T.F. Dupont, $L^{2}$-estimates for Galerkin methods for second order hyperbolic equations, SIAM J. Numer. Anal. 10 (1973), 880-889.

11. __ Mesh modification for evolution equations, Math. Comp. 39 (1982), 85-108.

12. B. Engquist and A. Majda, Absorbing boundary conditions for the numerical simulation of waves, Math. Comp. 31 (1977), 629-651.

13. T.J.R. Hughes, The finite element method: linear static and dynamic finite element analysis, Prentice-Hall, Englewood Cliffs, NJ, 1987.

14. G.M. Hulbert and T.J.R. Hughes, Space-time finite element methods for second-order hyperbolic equations, Comput. Methods Appl. Mech. Engrg. 84 (1990), 327-348. 
15. C. Johnson, Numerical solution of partial differential equations by the finite element method, Cambridge Univ. Press, Cambridge, 1987.

16. _ Discontinuous Galerkin finite element methods for second order hyperbolic problems, Comput. Methods Appl. Mech. Engrg. 107 (1993), 117-129.

17. K. Miller, Moving finite elements II, SIAM J. Numer. Anal. 18 (1981), 1033-1057.

18. K. Miller and R.N. Miller, Moving finite elements I, SIAM J. Numer. Anal. 18 (1981), 1019-1032.

19. J.A. Nitsche, Lineare Spline-funktionen und die Methode von Ritz für elliptische Randwertprobleme, Arch. Rational Mech. Anal. 36 (1970), 348-355.

20. A.H. Schatz, An observation concerning Ritz-Galerkin methods with indefinite bilinear forms, Math. Comp. 28 (1974), 959-962.

21. D.W. Sheen, Absorbing boundary conditions for wave transmissions, Technical Report \#159, Center for Applied Mathematics, Purdue University, West Lafayette, IN 47907, July 1991.

22. M.F. Wheeler, A priori $L^{2}$ error estimates for Galerkin approximations to parabolic partial differential equations, SIAM J. Numer. Anal. 10 (1973), 723-759.

23. D.Q. Yang, The mixed finite element methods with moving grids for parabolic problems, Math. Numer. Sinica 10 (1988), 266-271.

24. __ Grid modification for the wave equation with attenuation, Numer. Math. 67 (1994), 391-401.

25. __ Domain decomposition and grid modification for parabolic problems, (to appear).

Department of Mathematics, Purdue University, West Lafayette, Indiana 47907-1395

Department of Computer Science, Purdue University, West Lafayette, Indiana 479071398

E-mail address: yangd@math.purdue.edu

yangdQcs . purdue. edu 